\title{
Real-time correction of betatron tune ripples on a slowly extracted beam
}

\author{
D. Naito, ${ }^{*}$ Y. Kurimoto, R. Muto, T. Kimura, K. Okamura, T. Shimogawa, and M. Tomizawa \\ High Energy Accelerator Research Organization, Accelerator Laboratory, \\ Tokai, Ibaraki 319-1106, Japan
}

(Received 31 October 2018; published 17 July 2019)

\begin{abstract}
In the main ring (MR) of the Japan Proton Accelerator Research Complex (J-PARC), the power supplies of magnets induce large ripples in the betatron tunes. The tune ripples degrade the quality of the beam spill on the slowly extracted beam. To improve the spill quality, the J-PARC MR employed a feedback system using a beam spill monitor; however, the existing system could not correct the beam spills suitably, since the tune ripples were too large. We developed a new correction system using the information of magnet currents as an input of the correction. The new system consists of current monitors and a corrector quadrupole, and it allows us to correct the ripples in real time. Using this system, we successfully corrected the tune ripples for the first time in the J-PARC MR. The correction system improved the spill quality from $3 \%$ (without any correction) to $30 \%$ (with the new correction), whereas the quality using the present feedback system is $17.4 \%$.
\end{abstract}

DOI: 10.1103/PhysRevAccelBeams.22.072802

\section{INTRODUCTION}

The Japan Proton Accelerator Research Complex (J-PARC) is an accelerator facility that can provide highpower proton beams to the Material and Life Science Experimental Facility, the Hadron Experimental Facility (HEF), and the neutrino beam line for the long-baseline experiment, T2K. The J-PARC accelerator comprises a $400-\mathrm{MeV}$ proton linear accelerator (linac), a $3-\mathrm{GeV}$ rapidcycling synchrotron, and a $30-\mathrm{GeV}$ main ring (MR) [1]. The $\mathrm{MR}$ is a synchrotron that accelerates proton beams from 3 to $30 \mathrm{GeV}$; the accelerated beams are extracted fast to the neutrino beam line, or they are slowly extracted to the HEF that operates multiple high-energy and nuclear physics experiments [2-4]. During the slow extraction process, it is essential to achieve a high intensity together with a high extraction efficiency and as well as a high-quality "beam spill." The beam spill is a stream of particles slowly extracted from the MR during the extraction process as measured in the extraction beam line. The quality of the spill is mostly a function of the time structure in the beam intensity [5]. A perfect spill, that is a constant intensity as a function of time during the slow extraction, is ideal for data acquisition from particle detectors [6]. In the slow extraction beam line of the J-PARC MR, a beam power of $50 \mathrm{~kW}$, an extraction efficiency of $99.5 \%$, and a spill quality of $50 \%$ have been

\footnotetext{
*dnaito@post.j-parc.jp

Published by the American Physical Society under the terms of the Creative Commons Attribution 4.0 International license. Further distribution of this work must maintain attribution to the author(s) and the published article's title, journal citation, and DOI.
}

achieved [7]. With the future upgrades on the beam power, the quality of the beam spill should also be improved.

During the slow extraction process, the J-PARC MR moves the operation point of the betatron tune toward the region of the third integer resonance. The stability of the change rate in the betatron tune determines the quality of the beam spill. Therefore, the tune ripples, that are generated by the power supplies of the main magnets, affect the spill quality significantly. Even with several improvements in the power supplies [8,9], it was difficult to reduce the ripples of the power supplies sufficiently.

At various synchrotrons including the J-PARC MR, correction systems using a beam spill monitor have been employed to improve the quality of the beam spill [5, 10-12]. Since the signal from a spill monitor is modulated by its detector response, the correction from the spill signal is often imperfect. In particular, with the large tune ripples, the spill signals are insufficient for spill correction at the J-PARC MR. To improve the correction, the direct tune prediction is essential. In a quintessential way, the tune is measured using beam position monitors and excitation of the betatron oscillations; however, this method affects the slow extraction. Here, we introduced a new correction system that relies on the information of magnet currents that is the dominant source of the degradation of the spill quality. In this new system, the tune ripples were reconstructed in real time and corrected using a correction quadrupole magnet. A distinctive feature of the new system is the real-time and direct reconstruction of tune ripples without affecting the slow extraction.

Using our new system, we tested the reconstruction and correction of the tune ripples with improved spill quality. 
In Sec. II, the slow extraction at the J-PARC MR is described. The performance of the power supplies of the main magnets is also examined in this section. In Sec. III, our new correction system is presented. The results of the spill measurements using the proposed system is described in Sec. IV. Finally, the main conclusions are summarized in Sec. V.

\section{SLOW EXTRACTION AT THE J-PARC MR}

\section{A. Overview}

The J-PARC MR is a synchrotron having a circumference of $1567.5 \mathrm{~m}$ with a superperiod of 3 [1]. A layout of the MR is schematically shown in Fig. 1. The MR comprises three arc sections and three straight sections. Each arc section, which is $406.4 \mathrm{~m}$ long, comprises eight modules; each module consists of three FODO cells. Typically, a FODO cell consists of bending and quadrupole magnets, except for the second cell. The second cell does not include any bending magnets so that the transition energy becomes an imaginary value. Instead, it has sextupole magnets to correct the chromaticity $d \nu /(d p / p)$. A list of the magnets is shown in Table I. A group of the magnets, which is driven by a common power supply, is called a "family"; the power supplies are distributed in three buildings. Symbols "BM\#," where the \# indicates a number, represent the families of bending magnets. Symbols "Qxx" and "Sxx," where the Xx indicates characters, refer to the families of quadrupole and sextupole magnets, respectively. Each arc section includes 32 bending magnets, 57 quadrupole magnets, and 24 sextupole magnets. On the other hand, each straight section is $116 \mathrm{~m}$ long, and it contains three FODO cells, each of which consists of 15 quadrupole magnets. The straight section $\mathrm{A}$ is used for beam injection and collimation, section $\mathrm{B}$ is used for the slow extraction, and section $\mathrm{C}$ is used for the fast extraction and acceleration.

The kinetic energy of the proton beam during a single operation cycle is shown in Fig. 2. The operation cycle consists of four parts: injection, acceleration, debunching,

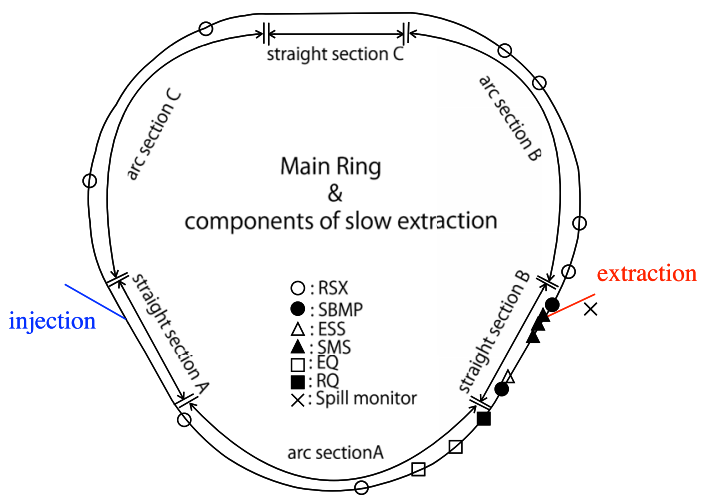

FIG. 1. A layout of the J-PARC MR with the principal components for the slow extraction. Symbols are explained in Secs. II A and II C.
TABLE I. Family of the main magnets.

\begin{tabular}{lclc}
\hline \hline Family & \# of magnets & \multicolumn{1}{c}{ Location } & Building \# \\
\hline BM1 & 16 & Arc section & 3 \\
BM2 & 16 & Arc section & 3 \\
BM3 & 16 & Arc section & 2 \\
BM4 & 16 & Arc section & 2 \\
BM5 & 16 & Arc section & 1 \\
BM6 & 16 & Arc section & 1 \\
QFN & 48 & Arc section & 2 \\
QDN & 48 & Arc section & 2 \\
QFX & 48 & Arc section & 3 \\
QDX & 27 & Arc section & 3 \\
QFR & 9 & Straight section & 1 \\
QFP & 6 & Straight section & 1 \\
QFT & 6 & Straight section & 1 \\
QFS & 6 & Straight section & 1 \\
QDR & 6 & Straight section & 1 \\
QDS & 6 & Straight section & 1 \\
QDT & 6 & Straight section & 1 \\
SFA & 24 & Arc section & 1 \\
SDA & 24 & Arc section & 1 \\
SDB & 24 & Arc section & 1 \\
\hline \hline
\end{tabular}

and slow extraction. Eight bunches of protons are injected into the MR within $0.14 \mathrm{~s}$ and accelerated from 3 to $30 \mathrm{GeV}$ in $1.4 \mathrm{~s}$. During the debunching process for $0.5 \mathrm{~s}$, the protons form a coasting beam by turning off the radiofrequency power. Finally, the beam is slowly extracted to the HEF for $2 \mathrm{~s}$.

Figure 3 illustrates the slow extraction in the normalized horizontal phase space. In Fig. 3, the separatrices indicated by a triangle define the boundaries between the stable and unstable region. An area of the stable region, $A_{s}$, is given by

$$
A_{s}=\frac{48 \sqrt{3} \pi^{2}}{S^{2}}\left(\nu_{r}-\nu_{x}\right)^{2}
$$

where $\nu_{x}$ represents the horizontal betatron tune, $\nu_{r}$ represents the tune of the resonance condition, and $S$ represents the normalized strength of the sextupole field.

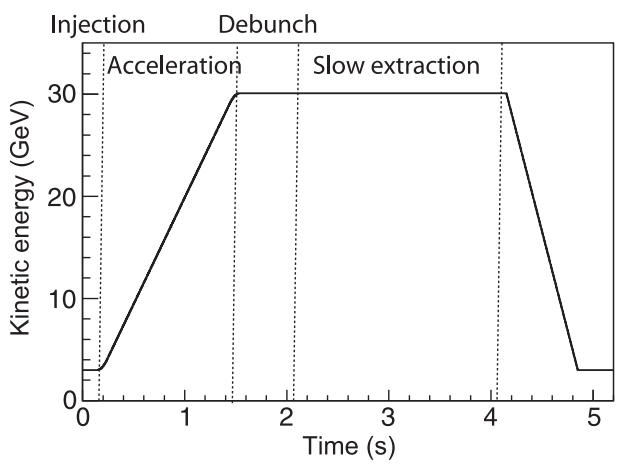

FIG. 2. The kinetic energy of the proton beam during a single operation cycle of the MR operating in the slow extraction mode. 


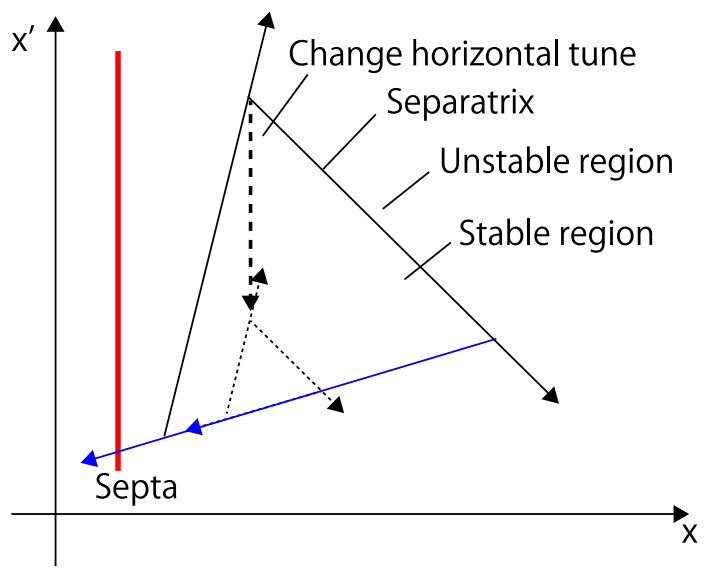

FIG. 3. The slow extraction scheme in the normalized horizontal phase space. The dashed lines represent the changes of the stable region. The blue line indicates the extraction separatrix.

To drive the $S$ term in Eq. (1), eight special sextupole magnets have been arranged, as indicated by RSX in Fig. 1. These sextupoles are grouped into two families so that they do not influence the chromaticity [5]. At the J-PARC MR, $\nu_{r}=67 / 3(=22.333)$ is employed for the slow extraction. By increasing the quadrupole fields of the QFN family, $\nu_{x}$ is gradually increased from 22.275 to $\nu_{r}$. In order to keep the orbit of the extracted beam, the quadrupole fields in the extraction section are not changed. When $\nu_{x}$ approaches the $\nu_{r}$, it causes a decrease in $A_{s}$, as shown in Eq. (1). This enables us to slowly squeeze almost all the protons from the ring to the outside of an electrostatic septum (ESS), where protons are separated by the electric field. Since the ESS is located at the dispersion-free region as well as the chromaticity is set to zero, the separatrices do not depend on particle momentum [13]. While increasing the tune, the displacement of the extraction separatrix, which is indicated by a blue line in Fig. 3, is compensated using a dynamic bump system, represented as "SBMP" in Fig. 1. With these two schemes, all protons intercept the ESS with the same angle $\left(\mathrm{X}^{\prime}\right)$ at an arbitrary time during the extraction process. This allows us to suppress the beam loss at the ESS significantly, resulting in achieving a high extraction efficiency of $99.5 \%$ [5]. Finally, an orbit of the extracted beam is bent by the magnetic septa (SMS) and delivered to the HEF.

\section{B. Current ripple of the power supplies}

The time structure of the beam spill, $S_{\text {spill }}(t)$, is described by

$$
S_{\text {spill }}(t)=\frac{d N}{d \nu_{x}} \cdot \frac{d \nu_{x}}{d t}
$$

where $N$ is the number of extracted protons. The change rate of the tune, $d \nu_{x} / d t$, is influenced by the tune ripples, and then it induces coarse extracted spills on the slow

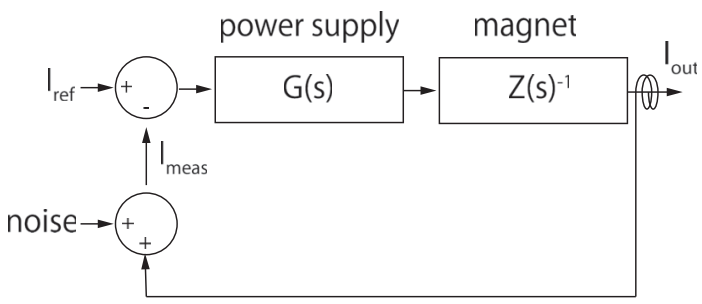

FIG. 4. Control diagram of the main magnet power supply.

extraction. The tune ripples are induced by fluctuations in the magnetic fields resulting from the current fluctuations at the power supplies of the bending and quadrupole magnets, as described below in Sec. III A. The control diagram of the power supplies is shown in Fig. 4. Symbols $I_{\text {meas }}$ and $I_{\text {ref }}$ represent the measured and reference currents, respectively. Figure 5 shows an example of the measured and reference currents, where the output current is regulated using the measured current. To evaluate the current ripples, we use the fractional current variation $\delta I$, which is defined by

$$
\delta I=\frac{I_{\text {meas }}-I_{\text {ref }}}{I_{\text {ref }}} .
$$

The measured current variation $\delta I$ in the frequency domain is shown in Fig. 6. Relatively large ripples are observed for the frequencies from 20 to $200 \mathrm{~Hz}$. In addition, few monochromatic peaks caused by rectification and semiconductor switching are also observed. The ripples in the low frequencies correspond to white noise, which is mixed into the current measurements, as shown in Fig. 4. Since both low-frequency and switching noises are not synchronized to the repetition cycle of beam operation, it is necessary to correct them in real time.

The contamination of the low-frequency noise over a large bandwidth is inevitable in the J-PARC MR, since the same control system and power supplies are used for both acceleration and extraction. In the case of acceleration, a

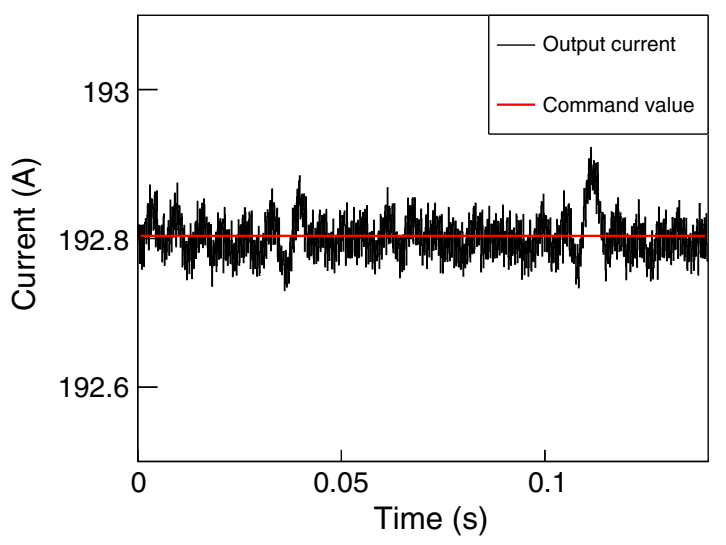

FIG. 5. Comparison between the measured and reference currents. 


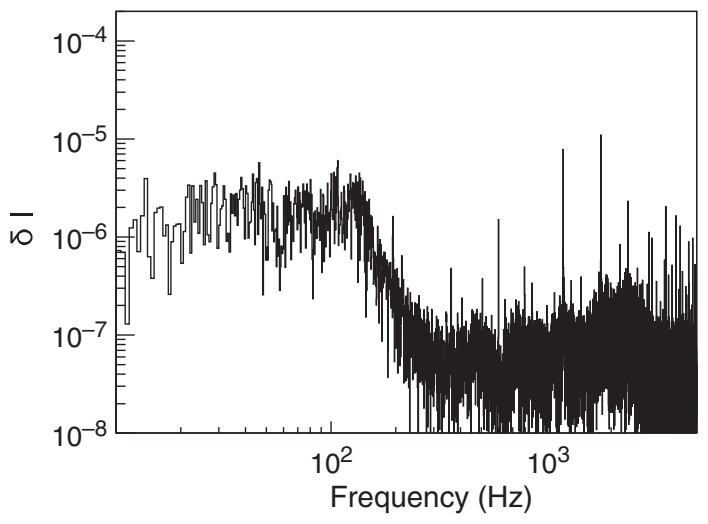

FIG. 6. The measured current variation $(\delta I)$ in the frequency domain.

large bandwidth is essential to minimize the tracking errors associated with the current regulation; the tracking error, where the output current deviates from the reference current, is induced at the beginning of acceleration when the control scheme for the current regulation switches [14]. Beam orbit distortion that is induced by these tracking errors must be minimized so that high-intensity proton beams are accelerated with small beam losses. On the other hand, in the case of the slow extraction, the large bandwidth induces inevitable current ripples at low frequencies, which is the dominant factor of the spill nonuniformity.

\section{Present correction system}

The present correction system consists of the spill feedback system and the transverse rf kicker [5]. A schematic of the present spill feedback system is shown in Fig. 7. The intensities of both the injected beam and the extracted spill are the main inputs of the feedback system. They are measured using a direct current transformer (DCCT) and a spill monitor, respectively. The spill monitor, which is a plastic scintillator plate $(5 \mathrm{~cm} \times 5 \mathrm{~cm} \times 5 \mathrm{~mm})$ mounted on a 2-inch photomultiplier tube (PMT), is placed

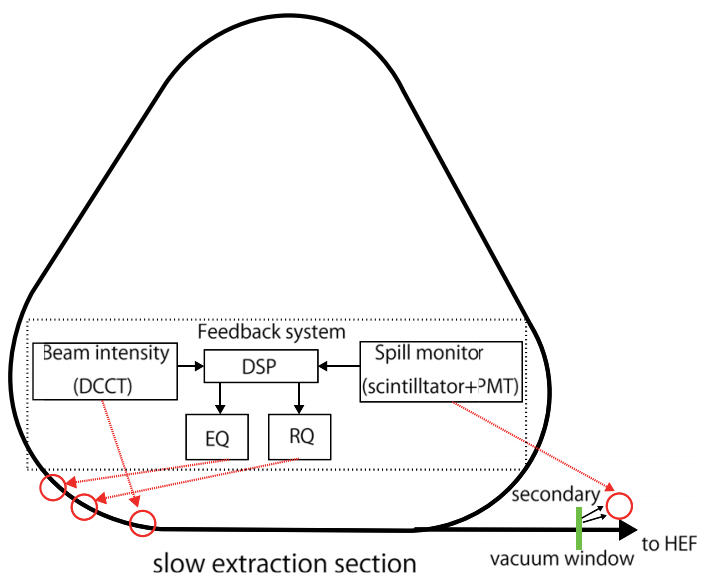

FIG. 7. Schematic of the present feedback system in the MR. at the extraction beam transport line. It detects secondary particles generated when the extracted protons hit a vacuum window. The window is $0.1-\mathrm{mm}$-thick aluminum, and the spill monitor is placed vertically to the beam line. Its solid angle to the secondary particles is $0.016 \mathrm{sr}$. The signal from the spill monitor is calibrated weekly by adjusting the PMT gain. The feedback system estimates the tune ripples using the spill signal. The correction based on the estimated tune is performed using two kinds of quadrupole magnets [15]. One is labeled as "EQ," and the other is labeled as "RQ." The EQ corrects the tune ripples for frequencies less than $20 \mathrm{~Hz}$, whereas the RQ corrects the tune ripples up to frequencies of several $\mathrm{kHz}$. These correction magnets can correct the full range of the current deviation shown in Fig. 6. However, the present feedback system cannot control the tune under the following conditions: (i) the tune is underestimated when the output of the spill monitor is saturated due to the extraordinary increase in the number of extracted protons per unit time, and (ii) the tune cannot be estimated when the output of the spill monitor is zero due to an unexpected disappearance of extracted protons.

Under these situations, the feedback system does not work effectively, because the estimated tune is different from the real tune. Because these situations occurred frequently due to large tune ripples, it has been difficult to achieve high spill quality. To address these difficulties, the direct tune prediction is essential.

\section{BETATRON TUNE PREDICTION AND CORRECTION SYSTEM}

We needed to correct the tune ripples by measuring or predicting them. However, tune measurement in real time is usually incompatible with slow extraction, because it requires one to kick the circulating beam [16]. Then, we have developed a new tune prediction and correction system using the current monitors of the power supplies. The concept of the system is shown in Fig. 8. The tune ripples are predicted using measured current variations $\delta I$. The predicted tune ripples are used to drive the correction quadrupole (RQ).

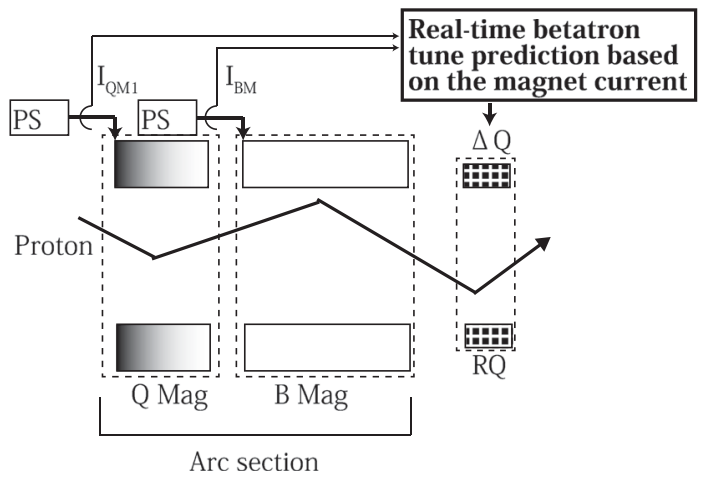

FIG. 8. Concept of the tune prediction and correction system. 


\section{A. Principle of the prediction}

The tune ripples $\Delta \nu_{\mathrm{QM}}(t)$, which are induced by the ripples of the quadrupole fields, are described as follows:

$$
\Delta \nu_{\mathrm{QM}}(t)=\frac{1}{4 \pi} \sum_{i=1}^{N_{\mathrm{QM}}} \beta_{i} \times \Delta K_{1, i}(t),
$$

where $N_{\mathrm{QM}}$ is the number of quadrupole magnets and $\Delta K_{1, i}(t)$ and $\beta_{i}$ are the ripples of the quadrupole field and the betatron amplitude function at the $i$ th quadrupole magnet, respectively. We used previously measured $\beta_{i}$ 's which were assumed to be constant; the changes of $\beta_{i}$ 's due to the current ripples were estimated to be less than 0.5\% using the Strategic Accelerator Design (SAD) [17] computer code.

The ripples of the dipole field also contribute to the tune ripples significantly. The dipole ripples distort the central orbit of the proton beam, and these orbital distortions at the sextupole magnets induce additional quadrupole fields. The tune ripples caused by the bending magnets $\left[\Delta \nu_{\mathrm{BM}}(t)\right]$ are described by the dipole ripples $\left[\Delta \theta_{\mathrm{BM}}(t)\right]$ and the sextupole fields $\left(K_{2}\right)$ as follows:

$$
\begin{aligned}
\Delta \nu_{\mathrm{BM}}(t)= & \frac{1}{8 \pi \sin \left(\pi \nu_{x}\right)} \sum_{i=1}^{N_{\mathrm{BM}}} \sum_{j=1}^{N_{\mathrm{SM}}} \Delta \theta_{\mathrm{BM}, i}(t) \times K_{2, j} \\
& \times \beta_{j}^{3 / 2} \beta_{i}^{1 / 2} \times \cos \left(-\pi \nu_{x}+\left|\phi_{i}-\phi_{j}\right|\right),
\end{aligned}
$$

where $\phi$ is the phase of the betatron oscillation, $N_{\mathrm{BM}}$ is the number of bending magnets, and $N_{\mathrm{SM}}$ is the number of sextupole magnets. The $\theta_{\mathrm{BM}}, K_{2}, \beta, \nu_{x}$, and $\phi$ were previously measured and assumed to be constant. The estimation of total tune ripple $\left[\Delta \nu_{\text {est }}(t)\right]$ is given by

$$
\Delta \nu_{\mathrm{est}}(t)=\Delta \nu_{\mathrm{QM}}(t)+\Delta \nu_{\mathrm{BM}}(t)
$$

Therefore, the tune ripples can be reconstructed based on real-time measurement of $\Delta \theta_{\mathrm{BM}}$ and $\Delta K_{1}$. It should be noted that $\Delta \theta_{\mathrm{BM}}$ or $\Delta K_{1}$ that belong to the same family are assumed to be the same value, and they are calculated from $\delta I$ assuming the following relations:

$$
\frac{\Delta \theta_{\mathrm{BM}}}{\theta_{\mathrm{BM}}}=\delta I_{\mathrm{BM}}, \quad \frac{\Delta K_{1}}{K_{1}}=\delta I_{\mathrm{QM}}
$$

We checked the correlation between the estimated $\Delta \theta_{\mathrm{BM}}$ and the measured one. An example of the correlation is shown in Fig. 9. The black line in Fig. 9 represents the fast Fourier transform (FFT) result of the $\Delta \theta_{\mathrm{BM}}$, which was calculated from Eq. (7) and $\delta I_{\mathrm{BM}}$. The red line represents the one obtained from the field measurement of the bending magnet using a pickup coil. The measured $\Delta \theta_{\mathrm{BM}}$ can be described by

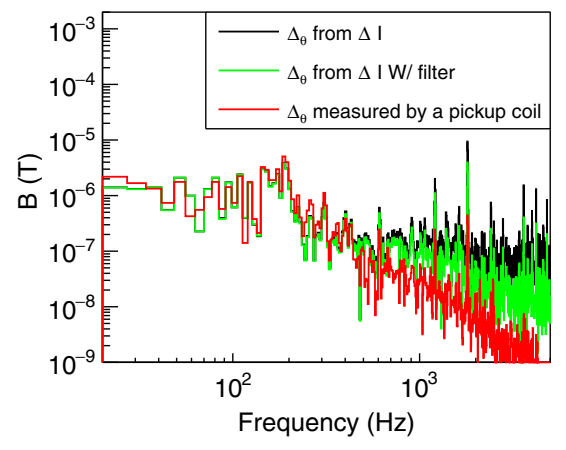

FIG. 9. Comparison between the measured dipole ripple (black line) and the one predicted by $\delta I$ (red line).

$$
\Delta \theta_{\mathrm{BM}}=\theta_{\mathrm{BM}} \times \delta I_{\mathrm{BM}}-C_{\mathrm{eddy}} \times \theta_{\mathrm{BM}} \times \frac{d}{d t}\left(\delta I_{\mathrm{BM}}\right) .
$$

The second term represents the effect of the eddy current induced on the beam ducts, and $C_{\text {eddy }}$ is determined by the material and the shape of the ducts. Therefore, measured ripples become smaller than the calculated ripples in the high-frequency domain. To reproduce the measured ripples, $\delta I$ is filtered using a low-pass filter with a cutoff frequency of $1 \mathrm{kHz}$, as indicated by the green line in Fig. 9. The correction using the filter is insufficient due to the limited performance of the field-programmable gate array (FPGA). However, the filtered $\delta I$ signal is adopted to the tune correction.

\section{B. Schematic of the prediction system}

A schematic of the tune prediction system [18] is shown in Fig. 10. The $\delta I$ 's of the power supplies which locate at the arc sections are used to reconstruct the tune ripples. The other $\delta I$ 's are not used, because they are connected to a small number of magnets, and, thus, they contribute little to the tune ripples. The $\delta I$ is digitized using an analog-todigital (AD) board that contains a 16-bit analog-to-digital converter (ADS8568 from Texas Instruments). The optical

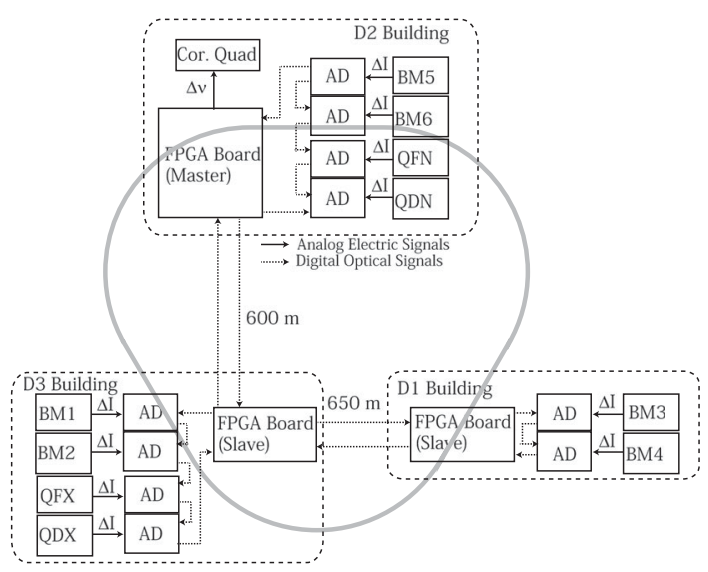

FIG. 10. Schematic of the tune prediction system. 
output of the AD board is connected to the FPGA board via a daisy chain. The sampling frequency and the system clock are $10 \mathrm{kHz}$ and $20 \mathrm{MHz}$, respectively. The FPGA board in buildings D1 or D3 collects the $\delta I$ and sends the data to the other FPGA boards. The FPGA board in building D2 collects the $\delta I$, and then it calculates the tune ripples using Eqs. (4)-(7). Finally, the reconstructed tune ripples are corrected by the RQ. We evaluated a proper current of RQ $\left(I_{\mathrm{RQ}}\right)$ to completely correct the tune by the relation

$$
I_{\mathrm{RQ}}(t)=\Delta \nu_{\mathrm{est}}(t) \times C_{\mathrm{RQ}},
$$

where the constant $C_{\mathrm{RQ}}$ was determined by the SAD code. The total delay of $I_{\mathrm{RQ}}$ relative to the output currents of the main magnets is less than $10 \mu \mathrm{s}$ and is sufficiently short to correct tune ripples in the frequencies less than $200 \mathrm{~Hz}$.

\section{Reconstruction of the tune ripples}

In estimating tune ripples $\left(\Delta \nu_{\text {est }}\right)$ from $\delta I$ 's, the effects of eddy currents are partially included, as explained in Sec. III A. However, most of them, especially some peaks induced by switching ripples of the power supply, remain uncorrected. Then, the real tune ripples $\left(\Delta \nu_{\text {real }}\right)$ are described using $\Delta \nu_{\text {est }}$, and the residual contribution due to the eddy currents $\left(\Delta \nu_{\text {eddy }}\right)$ are as follows:

$$
\Delta \nu_{\text {real }}=\Delta \nu_{\text {est }}-\Delta \nu_{\text {eddy }} .
$$

Measured tune ripples, which are almost the same as the $\Delta \nu_{\text {real }}$, are compared with the $\Delta \nu_{\text {est }}$. During the measurement, the proton beam was accelerated up to $30 \mathrm{GeV}$ and was not extracted. The circulating beam was kicked by the strip-line kicker to excite the betatron oscillation. The oscillations were measured by the beam position monitors. The FFT result of the measured position was used to reconstruct the tune ripples. The data for the FFT analysis were taken for $0.5 \mathrm{~s}$, and its starting time was set to be the same time as beginning of the slow extraction process. A comparison between the value of the measured ripples and the $\Delta \nu_{\text {est }}$ is shown in Fig. 11. The black line represents the FFT result of the measured ripples, whereas the red line represents the ripples calculated from Eqs. (4) and (5). The sampling frequency of the measured ripples was $5 \mathrm{kHz}$, whereas that of the calculated ripples was $10 \mathrm{kHz}$. In Fig. 11, the measured spectrum agreed with the estimated one in the frequencies lower than $200 \mathrm{~Hz}$. On the other hand, they differed in the frequencies higher than $600 \mathrm{~Hz}$; the measured spectrum contained white noise, whereas the estimated one contained uncorrected tune ripples $\left(\Delta \nu_{\text {eddy }}\right)$. Because the integrated amplitude of the high-frequency component was smaller than that of the low-frequency components in the estimated spectrum, we expected that the new system can compensate for the beam spill. As a basis for this, a comparison of the tune ripples

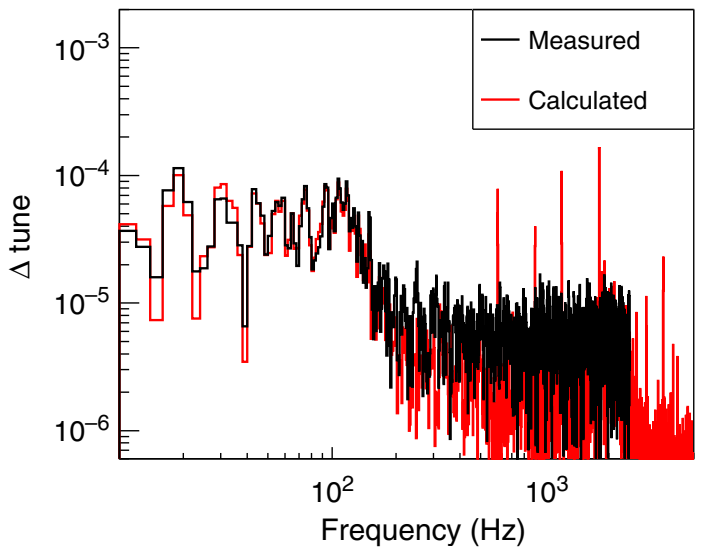

FIG. 11. Comparison between the measured and estimated tune ripples.

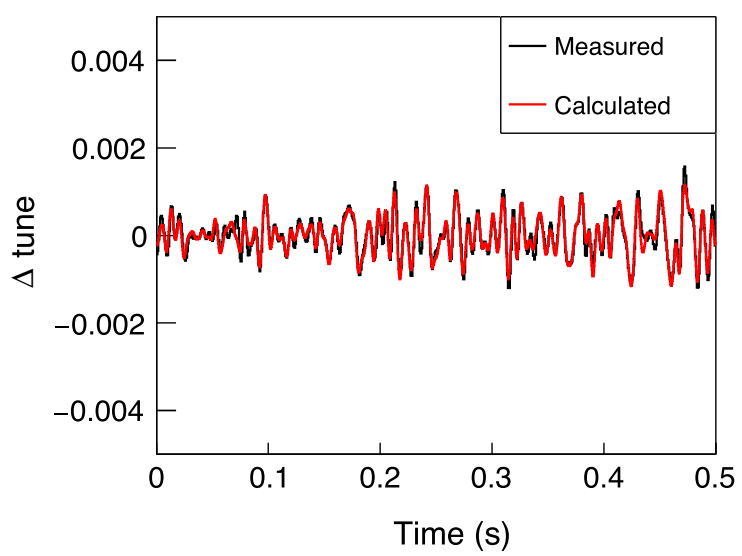

FIG. 12. Comparison of the tune ripples reconstructed from the frequency components between 20 and $200 \mathrm{~Hz}$.

reconstructed from the frequency components between 20 and $200 \mathrm{~Hz}$ is shown in Fig. 12. This plot indicates that the new prediction system can reconstruct the main features of the tune ripples, which are required for the slow extraction.

We applied the new correction system to the circulating beam. A comparison between the values of the measured ripples with and without the correction is shown in Fig. 13. The black and red lines represent the FFT results of the ripples without and with the correction, respectively. The other corrections were turned off during these measurements. The ripples decreased in the frequencies lower than $200 \mathrm{~Hz}$, whereas they increased for the frequencies higher than $600 \mathrm{~Hz}$, because the new correction system cannot correct the $\Delta \nu_{\text {eddy }}$ properly. Comparing the time variation of the tune ripples, the rms amplitude of the tune ripples under the correction was $80 \%$ of that without the correction.

\section{CORRECTION ON THE SLOW EXTRACTION}

We applied the new tune correction system to the slow extraction in June, 2018, and evaluated the signal of the 


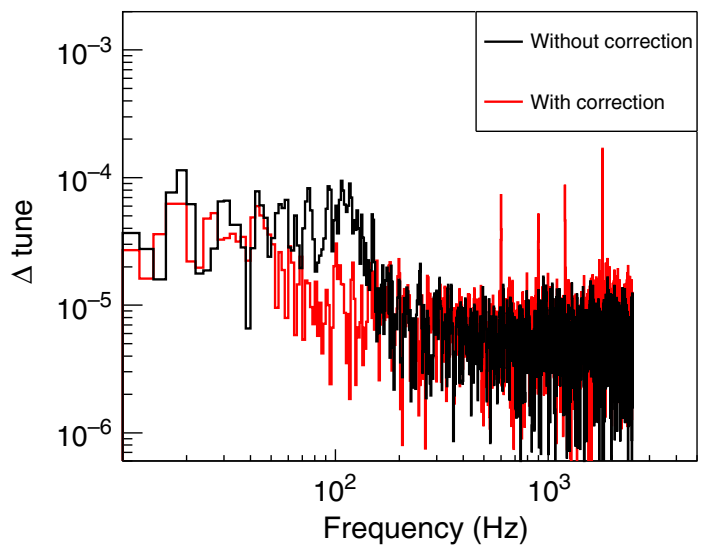

FIG. 13. Comparison of the tune ripples without and with the correction.

spill monitor and the spill quality. The conventional feedback system and the transverse rf, which were mentioned in Sec. II C, were turned off during these tests. The beam power was limited to $12 \mathrm{~kW}$, which was lower than that $(50 \mathrm{~kW})$ under the usual operation. This was needed to avoid excessive beam losses during slow extraction even when the new correction system did not work well. Both the beam optics and the beam orbit were adjusted to adapt the slow extraction without using our conventional correction systems. At this time, the extraction efficiency was $99.5 \%$, which was the same as that under usual operation.

The spill monitor signals are shown in Fig. 14. Events of the spikes in Fig. 14 are summarized by the histogram, as shown in Fig. 15. The black and red lines represent the results of the spill monitor signal without and with the correction, respectively. With the correction, large spikes in the spill monitor signal became less frequent, which indicates an improvement in the spill quality.

The FFTs of the spill monitor signal are shown in Fig. 16. The black and red lines represent the results

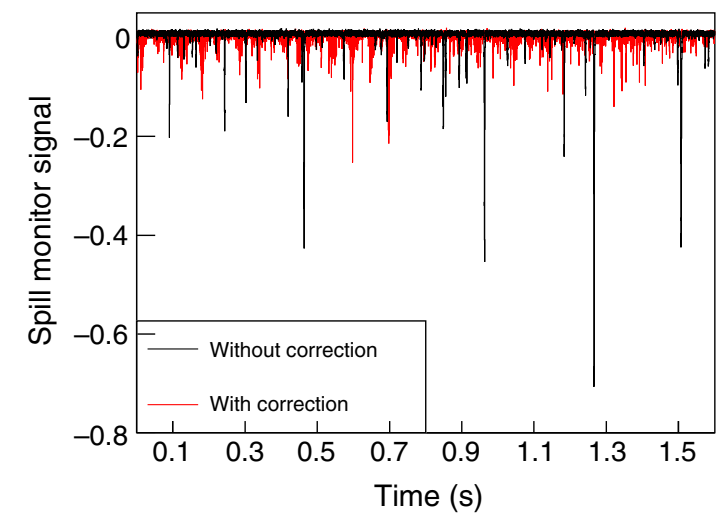

FIG. 14. Comparison of the spill signals without and with the new correction system.

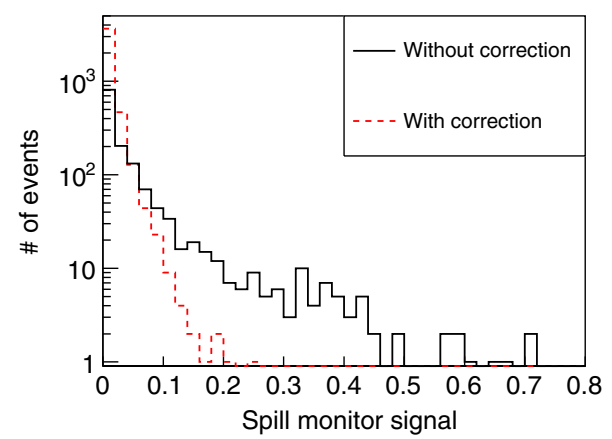

FIG. 15. The histogram showing events of the spikes in Fig. 14. Absolute values of the spill signals were used.

without and with the correction, respectively. The amplitudes of the signal in the frequencies between 20 and $200 \mathrm{~Hz}$ reduced by one order of magnitude, whereas the amplitude in the frequencies above $600 \mathrm{~Hz}$ is increased. The former fact accounts for the reduction in the number of large spikes, shown in Figs. 14 and 15, whereas the latter accounts for the increase in the number of small spikes in Fig. 14. Since the new correction system added $\Delta \nu_{\text {eddy }}$ to $\Delta \nu_{\text {real }}$, as explained in Sec. III C, the amplitudes of the spill monitor signal increased in the high-frequency domain.

The spill quality was evaluated using the duty factor (DF), which was calculated from the intensity of the beam spill $(I)$ as follows:

$$
\mathrm{DF}=\frac{\left\{\int I(t) d t\right\}^{2}}{\int d t \int I^{2}(t) d t}
$$

By applying the new correction system, the DF was improved from $3 \%$ to $30 \%$. As a comparison, the DF was also evaluated using only our conventional feedback in June, 2016. At a beam power of $40 \mathrm{~kW}$ and the extraction efficiency of $99.5 \%$, the DF was evaluated to be $17.4 \%$. These results imply that the newly developed system is superior to the conventional feedback.

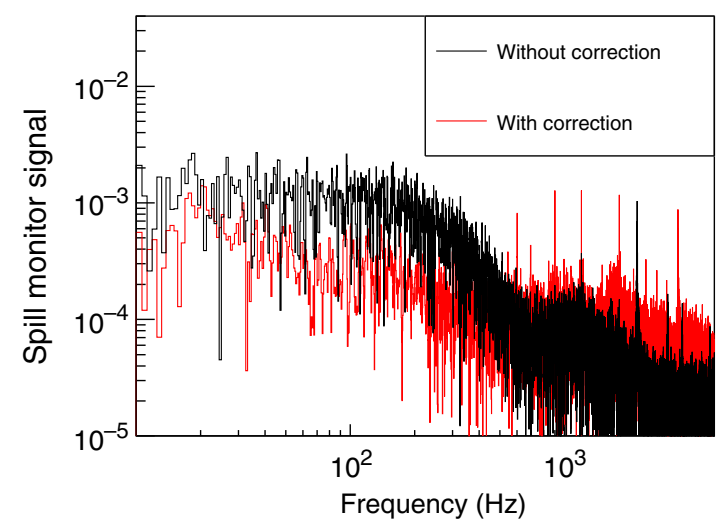

FIG. 16. FFT results of the spill signals. 


\section{CONCLUSION AND PROSPECT}

In the J-PARC MR, the power supplies of the magnets induce large ripples in the betatron tunes. The tune ripples degrade the beam spill quality on the slow extraction. To improve the spill quality, we developed a real-time tune prediction and correction system. The prediction part of the system consisted of eight current monitors and three FPGA boards. The betatron tune ripples were reconstructed from $\delta I$ 's in the frequencies between 20 and $200 \mathrm{~Hz}$. This system allows us to estimate the tune ripples in real time without interrupting the slow extraction process in the J-PARC MR. The reconstructed tune ripples were used to calculate the output current of the correction magnet. After applying the correction, the spill quality of the slow extraction improved from $3 \%$ to $30 \%$. This improvement was superior to that experienced with our conventional feedback system.

The achieved spill quality using the new system was still smaller than that achieved under usual operation of $50 \%$, because the transverse rf, which could improve the spill quality, was not turned on yet. In the future, we plan to tune the transverse rf together with the new correction system. It is also important to reduce the effect of the switching ripples in the correction output. This can be achieved by optimizing the low-pass filter for the output. In order to accomplish this task, it is necessary to measure and calculate the correlation between the current and the magnetic field of the main magnets. These approaches are promising for improving the spill quality further.

\section{ACKNOWLEDGMENTS}

The authors thank Professor Shogo Sakanaka for his valuable comments on this manuscript.

[1] KEK Report No. 2002-13, edited by Y. Yamazaki et al., 2002.

[2] J. K. Ahn et al. (KOTO Collaboration), Search for $K_{L} \rightarrow$ $\pi^{0} \nu \bar{\nu}$ and $K_{L} \rightarrow \pi^{0} X^{0}$ Decays at the J-PARC KOTO Experiment, Phys. Rev. Lett. 122, 021802 (2019).

[3] S. Ajimura, G. Beer, H. Bhang et al., A search for deeply bound kaonic nuclear states at J-PARC, Few-Body Syst. 54, 1195 (2013).

[4] T. Yamaga et al., Spectroscopic study of hyperon resonances below $\bar{K} N$ threshold via the $\left(K^{-}, n\right)$ reaction on deuteron, Jpn. Phys. Soc. Conf. Proc. 8, 021016 (2015).

[5] M. Tomizawa, Y. Arakaki, T. Kimura, R. Muto, S. Murasugi, K. Okamura, H. Sato, Y. Shirakabe, and E. Yanaoka, Slow extraction from the J-PARC main ring using a dynamic bump, Nucl. Instrum. Methods Phys. Res., Sect. A 902, 51 (2018).

[6] S. Su et al., The data acquisition system for the KOTO experiment and the RCE platform technology upgrade, IEEE Trans. Nucl. Sci. 64, 1338 (2017).
[7] M. Tomizawa, Y. Arakaki, T. Kimura, R. Muto, S. Murasugi, K. Okamura, Y. Shirakabe, and E. Yanaoka, Status and beam power ramp-up plans of the slow extraction operation at J-PARC Main Ring, in Proceedings of the 61st ICFA Advanced Beam Dynamics Workshop on High-Intensity and High-Brightness Hadron Beams (JACoW, Geneva, 2018), pp. 347-351.

[8] Y. Kurimoto, Y. Morita, S. Nakamura, and T. Shimogawa, Precise current control in accelerator magnets with a digital feedback system, IEEE Trans. Nucl. Sci. 61, 546 (2014).

[9] S. Nakamura, T. Shimogawa, Y. Morita, and Y. Kurimoto, Power supplies for main magnet of J-PARC Main Ring, in Proceedings of the 7th International Particle Accelerator Conference (JACoW, Geneva, 2016), pp. 3534-3536.

[10] M. Gyr, Proposal for a new servo-spill system: Power requirements for different configurations, Report No. CERN-SL-95-103-BT, CERN, 1995.

[11] M. Caldara, E. Rojatti, J. Bosser, G. M. A. Calvi, L. Lanzavecchia, A. Parravicini, and C. Viviani, Online spill intensity monitoring for improving extraction quality at $\mathrm{CNAO}$, in Proceedings of the 6th International Particle Accelerator Conference (JACoW, Geneva, 2015), pp. 907909.

[12] Y. C. Chen, Y. C. Feng, R. S. Mao, J. Shi, T. C. Zhao, M. Li, J. M. Dong, W. L. Li, and S. P. Li, Optimization and upgrade of slow extraction control system for HIRFL CSR main ring, in Proceedings of the 16th International Conference on Accelerator and Large Experimental Physics Control Systems (JACoW, Geneva, 2018), pp. 16631665.

[13] L. Badano et al., Proton-Ion Medical Machine Study (PIMMS) part 1, Report No. CERN-PS-99-010-DI, CERN, 2000, pp. 9-23.

[14] T. Shimogawa et al., Test demonstration of magnet power supply with the floating capacitor method, Jpn. Phys. Soc. Conf. Proc. 8, 012021 (2015).

[15] A. Kiyomichi, T. Adachi, A. Akiyama, S. Murasugi, R. Muto, H. Nakagawa, J. Odagiri, K. Okamura, Y. Sato, S. Sawada, Y. Shirakabe, H. Someya, K. H. Tanaka, M. Tomizawa, A. Toyoda, H. Sato, K. Mochiki, S. Onuma, T. Kimura, and K. Noda, Beam spill control for the J-PARC slow extraction, in Proceedings of the first International Particle Accelerator Conference (JACoW, Geneva, 2010), pp. 3933-3935.

[16] T. Toyama, D. Arakawa, M. Arinaga, K. Hanamura, H. Harada, Y. Hashimoto, S. Hatakeyama, N. Hayashi, S. Hiramatsu, S. Igarashi, S. Lee, H. Matsumoto, J. Odagiri, K. Sato, M. Tejima, M. Tobiyama, K. Yamamoto, and N. Yamamoto, Beam diagnostics at the first beam commissioning of the J-PARC MR, in Proceedings of the 23rd Particle Accelerator Conference, Vancouver, Canada, 2009 (JACoW, Geneva, 2010), pp. 1964-1966.

[17] SAD home page: http://acc-physics.kek.jp/SAD/.

[18] Y. Kurimoto, T. Shimogawa, and D. Naito, Real-time betatron tune correction with the precise measurement of magnet current, IEEE Trans. Nucl. Sci. (Early Access) 1 (2018). 